Mathematical Research Letters 5, 47-56 (1998)

\title{
SIMPLE LOOPS ON SURFACES AND THEIR INTERSECTION NUMBERS
}

\author{
FENG LUO
}

\begin{abstract}
Given a compact orientable surface $\Sigma$, let $\mathcal{S}(\Sigma)$ be the set of isotopy classes of essential simple loops on $\Sigma$. We determine a complete set of relations for a function from $\mathcal{S}(\Sigma)$ to $\mathbb{Z}$ to be a geometric intersection number function. As a consequence, we obtain explicit equations in $\mathbb{R}^{\mathcal{S}(\Sigma)}$ and $P\left(\mathbb{R}^{\mathcal{S}(\Sigma)}\right)$ defining Thurston's space of measured laminations and Thurston's compactification of the Teichmüller space. These equations are not only piecewise integral linear but also semi-real algebraic.
\end{abstract}

\section{Introduction}

Given a compact orientable surface $\Sigma=\Sigma_{g, r}$ of genus $g$ with $r(r \geq 0)$ boundary components, let $\mathcal{S}=\mathcal{S}(\Sigma)$ be the set of isotopy classes of essential simple loops on $\Sigma$. A function $f: \mathcal{S}(\Sigma) \rightarrow \mathbb{R}$ is called a geometric intersection number function, or simply geometric function if there is a measured lamination $m$ on $\Sigma$ so that $f(\alpha)$ is the measure of $\alpha$ in $m$. Geometric functions were introduced and studied by W. Thurston in his work on the classification of surface homeomorphisms and the compactification of the Teichmüller spaces ([FLP], $[\mathrm{HP}],[\mathrm{Th}])$. The space of all geometric functions under the pointwise convergence topology is homeomorphic to Thurston's space of measured laminations $\mathcal{M L}(\Sigma)$. Thurston showed that $\mathcal{M} L(\Sigma)$ is homeomorphic to a Euclidean space and $\mathcal{M L}(\Sigma)$ has a piecewise integral linear structure invariant under the action of the mapping class group. The projectivization of $\mathcal{M} L(\Sigma)$ is Thurston's boundary of the Teichmüller space. The purpose of this note is to announce a complete characterization of all geometric functions on $\mathcal{S}(\Sigma)$. As a consequence, both $\mathcal{M L}(\Sigma)$ and its projectivization are reconstructed explicitly in terms of an intrinsic combinatorial structure on $\mathcal{S}(\Sigma)$.

Theorem 1. Suppose $\Sigma$ is a compact orientable surface of negative Euler number. Then a function $f$ on $\mathcal{S}(\Sigma)$ is geometric if and only if for each incompressible subsurface $\Sigma^{\prime} \cong \Sigma_{1,1}$ or $\Sigma_{0,4}$, the restriction $\left.f\right|_{\mathcal{S}\left(\Sigma^{\prime}\right)}$ is geometric. Furthermore,

Received July 24, 1997.

This work was supported in part by the NSF. 
geometric functions on $\mathcal{S}\left(\Sigma_{1,1}\right)$ and $\mathcal{S}\left(\Sigma_{0,4}\right)$ are characterized by two homogeneous equations in an intrinsic $\left(\mathbb{Q} P^{1}, P S L(2, \mathbb{Z})\right)$ structure on $\mathcal{S}(\Sigma)$.

Recall that a subsurface $\Sigma^{\prime} \subset \Sigma$ is incompressible if each essential loop in $\Sigma^{\prime}$ is still essential in $\Sigma$. It is well known that if each boundary component of $\Sigma^{\prime}$ is essential in $\Sigma$, then $\Sigma^{\prime}$ is essential.

Geometric functions and measured laminations haven been studied from many different points of views. Especially, they are identified with height functions and horizontal foliations associated to holomorphic quadratic differentials on $\Sigma$ ([Ga], $[\mathrm{HM}],[\mathrm{Ker}])$. They are also related to the translation length functions of group action on $\mathbb{R}$-trees and the valuation theory ([Br], [CM], [MS], [Par], [Sk]). In [Bo1], measured laminations and hyperbolic metrics are considered as special cases of currents. As a consequence, Thurston's compactification is derived from a natural setting.

Our approach is combinatorial and is based on the notion of curve systems ([De], $[\mathrm{Th}])$. Recall that a curve system is a finite disjoint union of essential proper arcs and essential non-boundary parallel simple loops on the surface. Let $\mathcal{C} S(\Sigma)$ be the set of isotopy classes of curve systems on $\Sigma$. The space $\mathcal{C} S(\Sigma)$ was introduced by Dehn and rediscovered independently by Thurston. Dehn called the space the arithmetic field of the topological surface. Given two classes $\alpha$, $\beta$ in $\mathcal{C} S(\Sigma) \cup \mathcal{S}(\Sigma)$, their geometric intersection number $I(\alpha, \beta)$ is defined to be $\min \{|a \cap b|: a \in \alpha, b \in \beta\}$. The essential part of the proofs is to characterize those functions $f$ so that $f(\alpha)=I(\alpha, \beta)\left(=I_{\beta}(\alpha)\right)$ for some fixed $\beta \in \mathcal{C} S(\Sigma)$.
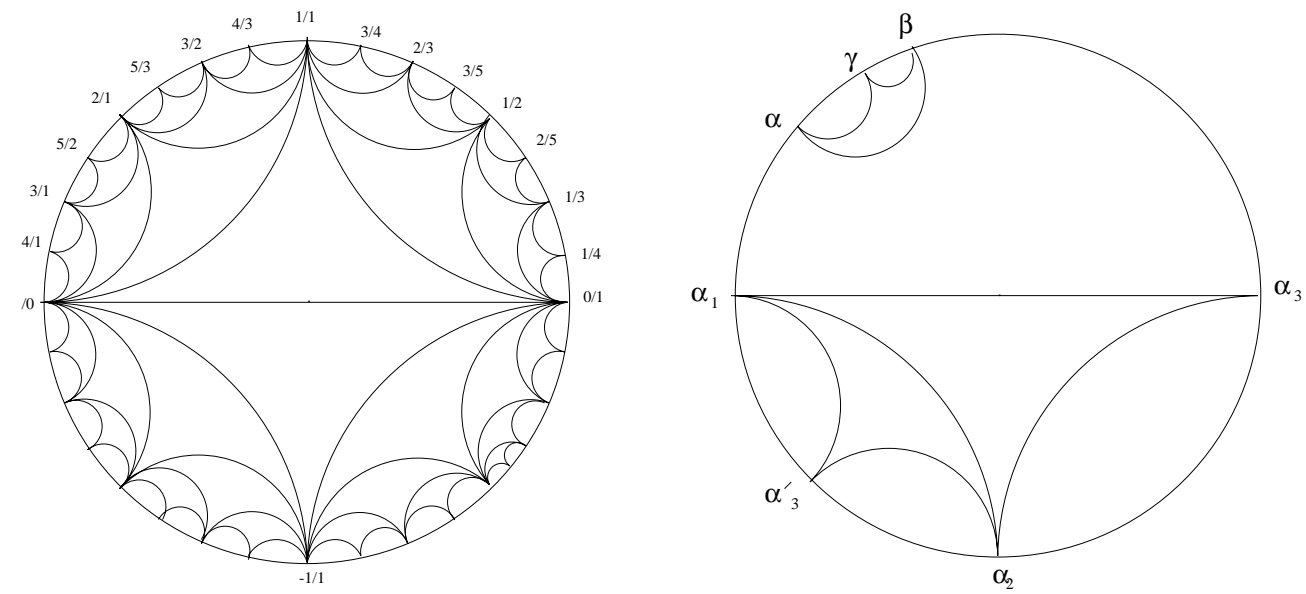

Figure 1

Given a surface $\Sigma$, let $\mathcal{S}^{\prime}(\Sigma)=\mathcal{C} S(\Sigma) \cap \mathcal{S}(\Sigma)$ be the set of isotopy classes of essential, non-boundary parallel simple loops in $\Sigma$. For surfaces $\Sigma=\Sigma_{1,0}, \Sigma_{1,1}$ and $\Sigma_{0,4}$, it is well known that there exists a bijection $\pi: \mathcal{S}^{\prime}(\Sigma) \rightarrow \mathbb{Q} P^{1}(=\hat{\mathbb{Q}})$ (the map sending a class to its slope) so that $\pi(\alpha)=p / q, \pi(\beta)=p^{\prime} / q^{\prime}$ satisfy $p^{\prime} q-p q^{\prime}= \pm 1$ if and only if $I(\alpha, \beta)=1$ (for $\Sigma_{1,0}, \Sigma_{1,1}$ ) and 2 (for $\Sigma_{0,4}$ ). 
See figure 1. We say that three distinct classes $\alpha, \beta, \gamma$ in $\mathcal{S}^{\prime}(\Sigma)$ form an ideal triangle if they correspond to the vertices of an ideal triangle in the modular relation under the map $\pi$. More generally, a $\left(\mathbb{Q} P^{1}, P S L(2, Z)\right)$ structure on a set $X$ is defined as follows. There is a maximal collection of charts $\{(U, \phi)\}$ where $U \subset X$ and $\phi: U \rightarrow \mathbb{Q} P^{1}$ is a bijection so that (a) the union of all these $U$ is the set $X$, and (b) if two charts $(U, \phi)$ and $(V, \psi)$ satisfy $U \cap V \neq \emptyset$, then the transition function $\phi \psi^{-1}$ is a restriction of an element in $\operatorname{PSL}(2, Z)$. For $X=\mathcal{S}^{\prime}(\Sigma)$, we take $U=\mathcal{S}^{\prime}\left(\Sigma^{\prime}\right)$ where $\Sigma^{\prime}$ is an incompressible subsurface homeomorphic to $\Sigma_{1,1}$ or $\Sigma_{0,4}$ and the map $\phi$ to be an orientation preserving slope map. This gives a $\left(\mathbb{Q} P^{1}, P S L(2, Z)\right)$ structure on $\mathcal{S}^{\prime}(\Sigma)$ which is invariant under the action of the group of orientation preserving homeomorphisms.

Theorem 2. (a) For surface $\Sigma_{1,1}$, a function $f: \mathcal{S} \rightarrow \mathbb{Z}_{\geq 0}$ is a geometric function $I_{\delta}$ with $\delta \in \mathcal{C} S(\Sigma)$ if and only if the following hold.

$$
f\left(\alpha_{1}\right)+f\left(\alpha_{2}\right)+f\left(\alpha_{3}\right)=\max _{i=1,2,3}\left(2 f\left(\alpha_{i}\right), f\left(\left[\partial \Sigma_{1,1}\right]\right)\right)
$$

where $\left(\alpha_{1}, \alpha_{2}, \alpha_{3}\right)$ is an ideal triangle.

$$
f\left(\alpha_{3}\right)+f\left(\alpha_{3}^{\prime}\right)=\max \left(2 f\left(\alpha_{1}\right), 2 f\left(\alpha_{2}\right), f\left(\left[\partial \Sigma_{1,1}\right]\right)\right)
$$

where $\left(\alpha_{1}, \alpha_{2}, \alpha_{3}\right)$ and $\left(\alpha_{1}, \alpha_{2}, \alpha_{3}^{\prime}\right)$ are two distinct ideal triangles.

$$
f\left(\left[\partial \Sigma_{1,1}\right]\right) \in 2 \mathbb{Z} .
$$

(b) For surface $\Sigma_{0,4}$ with $\partial \Sigma_{0,4}=b_{1} \cup b_{2} \cup b_{3} \cup b_{4}$, a function $f: \mathcal{S} \rightarrow \mathbb{Z}_{\geq 0}$ is a geometric function $I_{\delta}$ for some $\delta \in \mathcal{C} S(\Sigma)$ if and only if for each ideal triangle $\left(\alpha_{1}, \alpha_{2}, \alpha_{3}\right)$ so that $\left(\alpha_{i}, b_{s}, b_{r}\right)$ bounds a $\Sigma_{0,3}$ in $\Sigma_{0,4}$ the following hold.

$$
\Sigma_{i=1}^{3} f\left(\alpha_{i}\right)=\max _{1 \leq i \leq 3 ; 1 \leq s \leq 4}\left(2 f\left(\alpha_{i}\right), 2 f\left(b_{s}\right), \sum_{s=1}^{4} f\left(b_{s}\right), f\left(\alpha_{i}\right)+f\left(b_{s}\right)+f\left(b_{r}\right)\right)
$$

$$
f\left(\alpha_{3}\right)+f\left(\alpha_{3}^{\prime}\right)=\max _{1 \leq i \leq 2 ; 1 \leq s \leq 4}\left(2 f\left(\alpha_{i}\right), 2 f\left(b_{s}\right), \sum_{s=1}^{4} f\left(b_{s}\right), f\left(\alpha_{i}\right)+f\left(b_{s}\right)+f\left(b_{r}\right)\right)
$$

where $\left(\alpha_{1}, \alpha_{2}, \alpha_{3}\right)$ and $\left(\alpha_{1}, \alpha_{2}, \alpha_{3}^{\prime}\right)$ are two distinct ideal triangles,

$$
f\left(\alpha_{i}\right)+f\left(b_{s}\right)+f\left(b_{r}\right) \in 2 \mathbb{Z} .
$$

(c) The characterization of geometric functions $f: \mathcal{S}(\Sigma) \rightarrow \mathbb{R}_{\geq 0}$ for $\Sigma=\Sigma_{1,1}$ and $\Sigma_{0,4}$ is given by equations (1), (2) (for $\left.\Sigma_{1,1}\right)$ and (4), (5) $\left(\right.$ for $\left.\Sigma_{0,4}\right)$.

Theorem 2 is motivated by the torus case. In fact for the torus $\Sigma_{1,0}$, a function on $\mathcal{S}\left(\Sigma_{1,0}\right)$ is geometric if and only if it satisfies the triangle equality $f\left(\alpha_{1}\right)+$ $f\left(\alpha_{2}\right)+f\left(\alpha_{3}\right)=\max _{i=1,2,3}\left(2 f\left(\alpha_{i}\right)\right)$ and $f\left(\alpha_{3}\right)+f\left(\alpha_{3}^{\prime}\right)=\max \left(2 f\left(\alpha_{1}\right), 2 f\left(\alpha_{2}\right)\right)$. 
The equations (1),(2),(4) and (5) in theorem 2 are obtained as the degenerations of the trace identities for $S L(2, \mathbb{R})$ matrices. For instance, equations (1), (2) are the degenerations of $\operatorname{tr}(A) \operatorname{tr}(B) \operatorname{tr}(A B)=\operatorname{tr}^{2}(A)+\operatorname{tr}^{2}(B)+\operatorname{tr}^{2}(A B)-$ $\operatorname{tr}([A, B])-2$ and $\operatorname{tr}(A B) \operatorname{tr}\left(A^{-1} B\right)=\operatorname{tr}^{2}(A)+\operatorname{tr}^{2}(B)-\operatorname{tr}([A, B])-2$.

Several properties of the measured laminations spaces are reflected in the equations (1),(2),(4), and (5). For instance, since the equations are piecewise integral linear so that rational solutions are dense, one obtains Thurston's result that the space $\mathcal{M} L(\Sigma)$ has a piecewise integral linear structure and the rational multiples of the curve systems is a dense subset. On the other hand, the equations are also semi-real algebraic. Indeed, the space defined by $\sum_{i=1}^{k} x_{i}=$ $\max _{1 \leq j \leq l}\left(y_{j}\right)$ is semi-real algebraic since it is equivalent to: $\prod_{j=1}^{l}\left(\sum_{i=1}^{k} x_{i}-\right.$ $\left.y_{j}\right)=0$, and $\sum_{i=1}^{k} x_{i} \geq y_{j}$, for all $j$. This seems to indicate that the space $\mathcal{M} L(\Sigma)$ is semi-real algebraic.

Now given a surface $\Sigma_{g, r}$, Thurston showed that there exists a finite set $F$ consisting of $9 g+4 r-9$ elements in $\mathcal{S}(\Sigma)$ so that the map $\tau_{F}: \mathcal{M} L(\Sigma) \rightarrow \mathbb{R}_{\geq 0}^{F}$ sending $m$ to $\left.I_{m}\right|_{F}$ is an embedding ([FLP]). As a consequence of theorems 1,2 , we have,

Corollary. For surface $\Sigma_{g, r}$ of negative Euler number, there is a finite set $F$ consisting of $9 g+4 r-9$ elements in $\mathcal{S}(\Sigma)$ so that the map $\tau_{F}$ is an embedding whose image is a polyhedron defined by finitely many explicit integer coefficient polynomial equations and inequalities.

It is interesting to observe that the approach taken in this paper (also in [Lu1], [Lu2]) follows Grothendieck's general philosophy of the "Teichmüller tower" where the "generators" are the surfaces $\Sigma_{1,1}$ and $\Sigma_{0,4}$ and the "relations" are $\Sigma_{1,2}$ and $\Sigma_{0,5}$. (See [Sc]). From this point of view, it seems clear that the $\left(\mathbb{Q} P^{1}, P S L(2, \mathbb{Z})\right)$ modular structure is fundamental to the topology and geometry of surfaces and the modular structure plays a role of "local coordinates" on the set $\mathcal{S}(\Sigma)$.

\section{Sketch of the proof}

The necessity of the conditions in the theorems follows easily from the trace identities for $S L(2, \mathbb{R})$ matrices. For instance, to see equation (1), we use

$$
\operatorname{tr}(A) \operatorname{tr}(B) \operatorname{tr}(A B)=\operatorname{tr}^{2}(A)+\operatorname{tr}^{2}(B)+\operatorname{tr}^{2}(A B)-\operatorname{tr}([A, B])-2,
$$

where $A, B \in S L(2, \mathbb{R})$. If $A, B, A B$ correspond to three simple closed geodesics forming an ideal triangle in $\mathcal{S}$, then $\operatorname{tr}(A) \operatorname{tr}(B) \operatorname{tr}(A B)>0$ and $\operatorname{tr}([A, B])<$ 0 (see $[\mathrm{GiM}]$ for instance). In particular, we obtain $|\operatorname{tr}(A)||\operatorname{tr}(B)||\operatorname{tr}(A B)|=$ $\operatorname{tr}^{2}(A)+\operatorname{tr}^{2}(B)+\operatorname{tr}^{2}(A B)+|\operatorname{tr}([A, B])|-2$. The degeneration of it becomes $f(A)+f(B)+f(A B)=\max (2 f(A), 2 f(B), 2 f(A B), f([A, B]))$ which is equation $(1)$.

To show the sufficiency, we use induction on $\left|\Sigma_{g, r}\right|=3 g+r$. We first observe that the space of curve systems $\mathcal{C} S(\Sigma)$ has a natural multiplicative structure. 
The multiplicative structure induces an intrinsic combinatorial structure on $\mathcal{S}(\Sigma)$ which in turn determines the modular relation on $\mathcal{S}\left(\Sigma_{1,1}\right)$ and $\mathcal{S}\left(\Sigma_{0,4}\right)$. In terms of the multiplication, equations (2), (5) become iteration equations.

There are five steps involved in the proof. We single out step 1 (the multiplicative structure) in detail since it may have some interest in itself.

Assume that surfaces are oriented and connected and have negative Euler number. All surfaces drawn in the note have the right-hand orientation in the front face. A regular neighborhood of a submanifold $X$ is denoted by $N(X)$. The isotopy class of a curve system $c$ will be denoted by $[c]$. Suppose $f: \mathcal{C} S(\Sigma) \rightarrow \mathbb{R}$ is a function and $c$ is a curve system. We define $f(c)$ to be $f([c])$. In particular, $I(a, b)=I([a],[b])$. For simplicity, we say that a function $f: \mathcal{S} \rightarrow \mathbb{R}$ is a local geometric function if its restriction to $\mathcal{S}\left(\Sigma^{\prime}\right)$ is geometric for each incompressible subsurface $\Sigma^{\prime} \cong \Sigma_{1,1}$ and $\Sigma_{0,4}$. The goal is to show that a local geometric function is a geometric function.

Step 1. A multiplicative structure on $\mathcal{C} S(\Sigma)$ and the modular relation.

Suppose $a$ and $b$ are two arcs in $\Sigma$ intersecting transversely at one point $P$. Then the resolution of $a \cup b$ at $P$ from $a$ to $b$ is defined as follows. Take any orientation on $a$ and use the orientation on $\Sigma$ to determine an orientation on $b$. Then resolve the intersection according to the orientations. The resolution is independent of the choice of the orientation on $a$. See figure 2 (a).

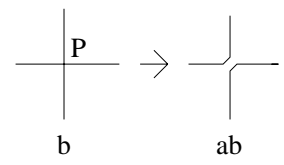

(a)

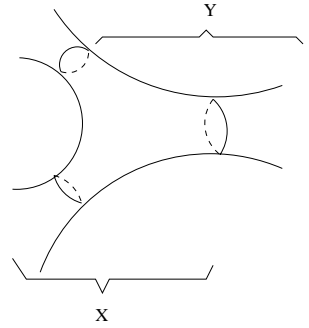

(b)

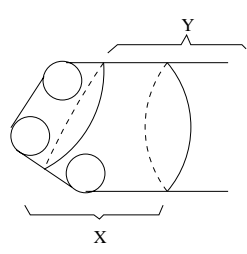

(c)

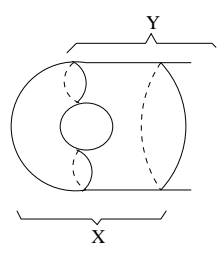

Figure 2

Given two curve systems $a, b$ on $\Sigma$ with $|a \cap b|=I(a, b)$, the multiplication $a b$ is defined to be the disjoint union of simple loops and arcs obtained by resolving all intersection points from $a$ to $b$. It can be shown that $a b$ is again a curve system whose isotopy class depends only on the isotopy classes of $a, b$. Given $\alpha, \beta \in \mathcal{C} S(\Sigma)$, we define $\alpha \beta=[a b]$ where $a \in \alpha, b \in \beta$ so that $|a \cap b|=I(a, b)$. The following theorem establishes the basic properties of the multiplication.

Let $\mathcal{C} S_{0}(\Sigma)$ be the subset of $\mathcal{C} S(\Sigma)$ consisting of isotopy classes of curve systems which contain no arcs.

Theorem 3. The multiplication $\mathcal{C} S(\Sigma) \times \mathcal{C} S(\Sigma) \rightarrow \mathcal{C} S(\Sigma)$ sends $\mathcal{C} S_{0}(\Sigma) \times$ $\mathcal{C} S_{0}(\Sigma)$ to $\mathcal{C} S_{0}(\Sigma)$ and satisfies the following properties. 
(a) It is preserved by the action of the orientation preserving homeomorphisms.

(b) If $I(\alpha, \beta)=0$, then $\alpha \beta=\beta \alpha$. Conversely, if $\alpha \beta=\beta \alpha$ and $\alpha \in \mathcal{C} S_{0}(\Sigma)$, then $I(\alpha, \beta)=0$.

(c) If $\alpha \in \mathcal{C} S_{0}(\Sigma), \beta \in \mathcal{C} S(\Sigma)$, then $I(\alpha, \alpha \beta)=I(\alpha, \beta \alpha)=I(\alpha, \beta)$ and $\alpha(\beta \alpha)=(\alpha \beta) \alpha$. If in addition each component of $\alpha$ intersects $\beta$, then $\alpha(\beta \alpha)=$ $\beta$.

(d) If $\left[c_{i}\right] \in \mathcal{C} S(\Sigma)$ so that $\left|c_{i} \cap c_{j}\right|=I\left(c_{i}, c_{j}\right)$ for $i, j=1,2,3, i \neq j, \mid c_{1} \cap$ $c_{2} \cap c_{3} \mid=0$, and there is no contractable region in $\Sigma-\left(c_{1} \cup c_{2} \cup c_{3}\right)$ bounded by three arcs in $c_{1}, c_{2}, c_{3}$, then $\left[c_{1}\right]\left(\left[c_{2}\right]\left[c_{3}\right]\right)=\left(\left[c_{1}\right]\left[c_{2}\right]\right)\left[c_{3}\right]$.

(e) For any positive integer $k,\left(\alpha^{k} \beta^{k}\right)=(\alpha \beta)^{k}$.

(f) If $\alpha$ is the isotopy class of a simple closed curve, then the positive Dehn twist along $\alpha$ sends $\beta$ to $\alpha^{k} \beta$ where $k=I(\alpha, \beta)$.

It follows from the definition that $I(\alpha, \gamma)+I(\beta, \gamma) \geq I(\alpha \beta, \gamma)$. Futhermore, theorem (c) implies a stronger result that $I(\alpha \beta, \gamma)+I(\alpha, \gamma) \geq I(\beta, \gamma)$ when $\alpha, \beta \in C S_{0}(\Sigma)$. Indeed, $I(\alpha \beta, \gamma)+I(\alpha, \gamma) \geq I((\alpha \beta) \alpha, \gamma) \geq I\left(\beta \delta^{2}, \gamma\right) \geq I(\beta, \gamma)$ where $\delta$ consists of components of $\alpha$ which are disjoint from $\beta$.

Remark. The multiplication on $\mathcal{C} S(\Sigma)$ seems to be related to the extension of Thurston's earthquakes to $\mathcal{M L}(\Sigma)$. See [Bo2], [Pap] for more information.

The modular relations on $\mathcal{S}\left(\Sigma_{1,1}\right)$ and $\mathcal{S}\left(\Sigma_{0,4}\right)$ are defined in terms of the multiplication as follows. Call two elements $\alpha, \beta \in \mathcal{S}(\Sigma)$ orthogonal, denoted by $\alpha \perp \beta$, if $I(\alpha, \beta)=1$; and pseudo-orthogonal, denoted by $\alpha \perp_{0} \beta$, if $I(\alpha, \beta)=2$ so that their algebraic intersection number is zero. Suppose $\alpha \perp \beta$ or $\alpha \perp_{0} \beta$. Take $a \in \alpha, b \in \beta$ so that $|a \cap b|=I(\alpha, \beta)$. Then $N(a \cup b) \cong \Sigma_{1,1}$ if $\alpha \perp \beta$ and $N(a \cup b) \cong \Sigma_{0,4}$ if $\alpha \perp_{0} \beta$. It follows from the definition that $\alpha \beta \perp \alpha, \beta$ if $\alpha \perp \beta$, and $\alpha \beta \perp_{0} \alpha, \beta$ if $\alpha \perp_{0} \beta$. Thus three distinct elements $\alpha, \beta, \gamma \in \mathcal{S}^{\prime}\left(\Sigma_{1,1}\right)$ (resp. $\mathcal{S}^{\prime}\left(\Sigma_{0,4}\right)$ ) form an ideal triangle if and only if $\alpha \perp \beta$ (resp. $\alpha \perp_{0} \beta$ ) and $\gamma \in\{\alpha \beta, \beta \alpha\}$. In particular the distinct ideal triangles in equations (2), (5) in theorem 2 are $\left(\alpha_{1}, \alpha_{2}, \alpha_{1} \alpha_{2}\right)$ and $\left(\alpha_{1}, \alpha_{2}, \alpha_{2} \alpha_{1}\right)$ where $\alpha_{1} \perp \alpha_{2}$ or $\alpha_{1} \perp_{0} \alpha_{2}$ (and in fact $\left(\alpha_{1}, \alpha_{2}, \alpha_{1} \alpha_{2}\right)$ is positively oriented). If $\alpha \perp \beta$ or $\alpha \perp_{0} \beta$, we define $\alpha^{-n} \beta=\beta \alpha^{n}$ for $n \in \mathbb{Z}_{>0}$. It follows from theorem $3(\mathrm{c})$ that $\alpha^{n}\left(\alpha^{m} \beta\right)=\alpha^{n+m} \beta$ for $n, m \in \mathbb{Z}$.

For $\Sigma=\Sigma_{1,1}$ or $\Sigma_{0,4}$, we can find the explicit bijection $\pi$ from $\mathcal{S}^{\prime}(\Sigma)$ to $\hat{\mathbb{Q}}$ as follows. Take $\alpha, \beta$ in $\mathcal{S}^{\prime}(\Sigma)$ so that $\alpha \perp \beta$ or $\alpha \perp_{0} \beta$. Then each $\gamma$ in $\mathcal{S}^{\prime}(\Sigma)$ can be expressed uniquely as $\alpha^{p} \beta^{q}$ where $q \in \mathbb{Z}_{\geq 0}, p \in \mathbb{Z}$ and $p, q$ are relatively prime. Define $\pi(\gamma)=p / q$ from $\mathcal{S}^{\prime}(\Sigma)$ to $\hat{\mathbb{Q}}$. Then $\pi\left(\gamma_{i}\right)=p_{i} / q_{i}, i=1$, 2 , satisfy $p_{1} q_{2}-p_{2} q_{1}= \pm 1$ if and only if $\gamma_{1} \perp \gamma_{2}$ or $\gamma_{1} \perp_{0} \gamma_{2}$.

Given two simple loops $a, b$, we use $a \perp b$ to denote $|a \cap b|=I(a, b)=1$, and use $a \perp_{0} b$ to denote $|a \cap b|=I(a, b)=2$ and $[a] \perp_{0}[b]$.

Step 2. Theorem 2 for surfaces $\Sigma_{1,1}$ and $\Sigma_{0,4}$.

The proof goes as follows. We first show the theorem for integer valued functions $f$ on $\mathcal{S}$. For integer valued $f$, there is an ideal triangle in $\mathcal{S}^{\prime}(\Sigma)$ so that the sum of the values of $f$ at the three vertices is minimal. This triangle is the 
key for the construction of the curve system $m \in C S(\Sigma)$ so that $f=I_{m}$. For general real valued function $f$, we show that $f$ can be approximated by rational valued functions which solve equations $(1),(2),(4),(5)$.

Step 3. A gluing lemma.

Suppose $\Sigma^{\prime}$ is an incompressible subsurface of $\Sigma$. We define the restriction $\operatorname{map} R\left(=R_{\Sigma^{\prime}}^{\Sigma}\right): \mathcal{C} S(\Sigma) \rightarrow \mathcal{C} S\left(\Sigma^{\prime}\right)$ as follows. Given $\alpha$ in $\mathcal{C} S(\Sigma)$, take $a \in \alpha$ so that $\left|a \cap \partial \Sigma^{\prime}\right|=I\left(a, \partial \Sigma^{\prime}\right)$ and $a \cap \Sigma^{\prime}$ contains no component parallel into $\partial \Sigma^{\prime}$. We define $R(\alpha)=\left[\left.a\right|_{\Sigma^{\prime}}\right]\left(:=\left.\alpha\right|_{\Sigma^{\prime}}\right)$. The restriction map is evidently well defined and furthermore if $X \subset Y \subset Z$ are incompressible subsurfaces, then $R_{X}^{Z}=R_{X}^{Y} R_{Y}^{Z}$.

Lemma 1 (Gluing along a 3-holed sphere). Suppose $X$ and $Y$ are connected incompressible subsurfaces in $\Sigma$ so that $\Sigma=X \cup Y$ and $X \cap Y \cong \Sigma_{0,3}$ as in figure 2(b). Then for any two elements $\alpha_{X} \in \mathcal{C} S(X), \alpha_{Y} \in \mathcal{C} S(Y)$ with $\left.\alpha_{X}\right|_{X \cap Y}=\left.\alpha_{Y}\right|_{X \cap Y}$, there is a unique element $\alpha \in \mathcal{C} S(\Sigma)$ so that $\left.\alpha\right|_{X}=\alpha_{X}$ and $\left.\alpha\right|_{Y}=\alpha_{Y}$.

This lemma also holds for measured laminations.

Step 4. Reduction to surfaces $\Sigma_{0,5}$ and $\Sigma_{1,2}$.

If $|\Sigma| \geq 5$, we decompose $\Sigma=X \cup Y$ where $X \cap Y \cong \Sigma_{0,3}$ and $X \cong \Sigma_{1,1}$ if $g \geq 1$ and $X \cong \Sigma_{0,4}$ if $g=0$ (see figure 2(c)). Suppose $f: \mathcal{S}(\Sigma) \rightarrow \mathbb{R}$ is a local geometric function. Then the restrictions $\left.f\right|_{\mathcal{S}(X)}$ and $\left.f\right|_{\mathcal{S}(Y)}$ are again local geometric functions. By the induction hypothesis and the gluing lemma, we find a measured lamination $m \in \mathcal{M} L(\Sigma)$ so that $\left.f\right|_{\mathcal{S}(X) \cup \mathcal{S}(Y)}=\left.I_{m}\right|_{\mathcal{S}(X) \cup \mathcal{S}(Y)}$. The goal is to show that $f=I_{m}$ using the equations (1),(2),(4),(5).

Let $d=\partial X \cap \operatorname{int}(\Sigma)$ be the separating simple loop in $\Sigma$. We show the following key lemma. (This is the part which is substantially different from and much more difficult than the case of Teichmüller space).

Lemma 2. If $f(\alpha)=I_{m}(\alpha)$ for all $\alpha \in \mathcal{S}$ so that $I(\alpha, d) \leq 2$, then $f=I_{m}$.

The proof is by induction on the complexity $(|\Sigma|, I(\alpha, d))$ in the lexicographic order. If $k=I(\alpha, d) \geq 3$, choose $x \in \alpha$ so that $|x \cap d|=k$. Fix an orientation on $x$. There are three possibilities which may occur for $x \cup d$ as listed in figure 3 where the intersection points $P_{1}, . ., P_{4}$ are adjacent along $d$. 


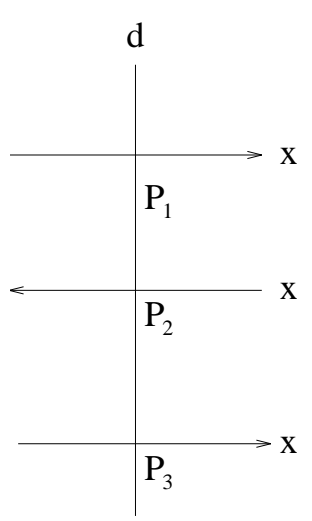

case (i)

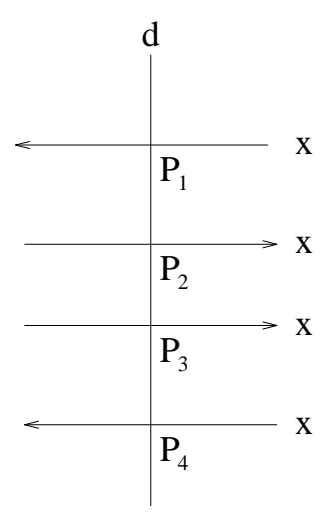

case (ii)

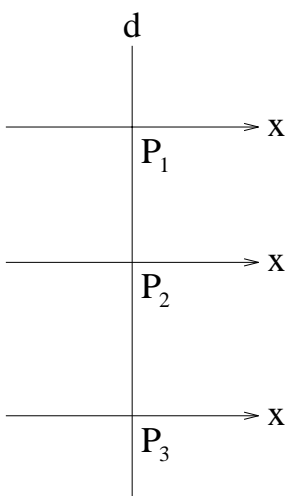

case (iii)

Figure 3

In case (i), we use a Lickorish's type lemma (see the lemma in [Lu2]) to produce a reduction of $\alpha$. In case (ii), there are six subcases need to be considered due the possible connections of the four horizontal arcs. Applying the Lickorish's type lemma twice will produce a reduction. In case (iii), we find two simple loops $p \perp q$ as shown in figure 4(a) so that $p q \cong x,|q \cap d|,|q p \cap d|<|x \cap d|$, and more importantly $|p \cap d|<\frac{1}{2}|x \cap d|$. By the induction hypothesis, $f$ and $I_{m}$ have the same values at $p, q, q p$. Now equation (2) for $p \perp q$ states that $f(x)+f(q p)=\max (2 f(p), 2 f(q), f(b))$ where $b=\partial N(p \cup q)$. Thus $f(x)=I_{m}(x)$ will follow from $f(b)=I_{m}(b)$. To prove this, we cut the surface $\Sigma$ open along $p$ (more precisely along $p^{\prime} \cong p$ with $\left|p^{\prime} \cap d\right|=I\left(p^{\prime}, d\right)$ ) to obtain a simpler surface $\Sigma^{\prime}$ which contains the curve $b$. Use the induction hypothesis and the following fact to conclude that $f=I_{m}$ on $\mathcal{S}\left(\Sigma^{\prime}\right)$.

Fact. Suppose $F$ is a surface of negative Euler number and $c$ is a curve system on $F$ consisting of $N$ arcs. Then each measured lamination $m \in \mathcal{M} L(F)$ is determined by its intersection numbers with $\beta \in \mathcal{S}(F)$ so that $I(\beta, c) \leq 2 N$.

Take $F=\Sigma^{\prime}, c=d \cap \Sigma^{\prime}$ and use $|p \cap d|<\frac{1}{2}|x \cap d|$. The result follows.

It remains to show $f(\alpha)=I_{m}(\alpha)$ for $\alpha \perp_{0}[d]$. Take $x \in \alpha$ so that $|x \cap d|=2$. Consider $\Sigma^{\prime}=N(x \cup X)$ which is homeomorphic either to $\Sigma_{1,2}$ or $\Sigma_{0,5}$. Thus to finish the proof, by considering $\left.f\right|_{\mathcal{S}\left(\Sigma^{\prime}\right)}$, it suffices to prove theorem 1 for surfaces $\Sigma_{1,2}$ and $\Sigma_{0,5}$.

Step 5. Theorem 1 for surfaces $\Sigma_{0,5}$ and $\Sigma_{1,2}$.

To show theorem 1 for $\Sigma_{0,5}$, we again follow the reduction step 4 . Now use the equations (4),(5) and the following pentagon relations on $\mathcal{S}\left(\Sigma_{0,5}\right): a_{i} a_{i+1} a_{i+2}=$ $a_{i+3} a_{i+4}$ where $a_{i}^{\prime} s$ are as shown in figure $4(\mathrm{~b})$. The surface $\Sigma_{1,2}$ is more complicated and the proof uses the five curves $a_{i}^{\prime} s$ as shown in figure 4(b). Note that the set of curves $\left\{a_{1}, \ldots, a_{5}\right\}$ is completely characterized up to homeomorphism by the property that $\left|a_{i} \cap a_{j}\right|=0$ for $|i-j| \geq 2$ (see [Lu3]). 
This finishes the sketch of the proof.

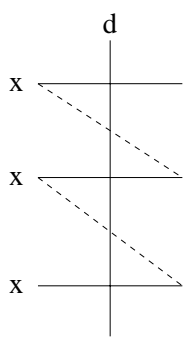

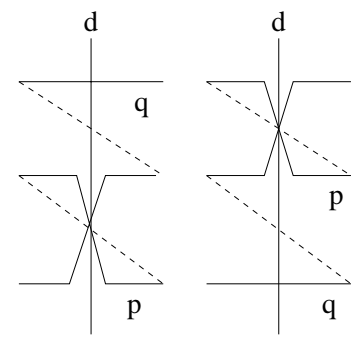

Choose $\mathrm{p}$ to be one of above

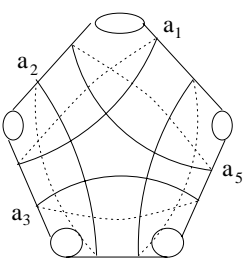

$\mathrm{a}_{4}$

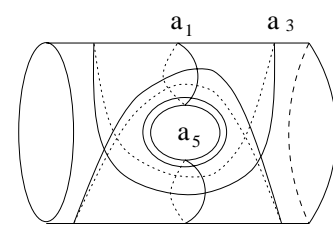

$\mathrm{a}_{2} \quad \mathrm{a}_{4}$

(a)

Figure 4

\section{Acknowledgement}

I would like to thank F. Bonahon, M. Freedman, X. S. Lin, and Y. Minsky for discussions.

\section{References}

[Bo1] F. Bonahon, The geometry of Teichmüller space via geodesic currents, Invent. Math. 92 (1988), 139-162.

[Bo2] - Earthquakes on Riemann surfaces and on measured geodesic laminations, Trans. Amer. Math. Soc. 330 (1992), 69-95.

[Br] G. W. Brumfiel, The real spectrum compactification of Teichmüller space, Contemp. Math., 74, Amer. Math. Soc., 1988, pp. 51-75.

[CM] M. Culler and J. Morgan, Group actions on R-trees, Proc. London Math. Soc. 55 (1987), 571-604.

[FLP] A. Fathi, F. Laudenbach and V. Poenaru, Travaux de Thurston sur les surfaces, Astérisque ,66-67, Société Mathématique de France, 1979.

[Ga] F. Gardiner, Teichmüller theory and quadratic differentials, John Wiley \& Sons, New York, 1987.

[GiM] J. Gilman and B. Maskit, An algorithm for 2-generator Fuchsian groups, Michigan Math. J. 38 (1991)), 13-32.

[HM] J. Hubbard and H. Masur, Quadratic differentials and foliations, Acta Math. 142 (1979), 221-274.

[HP] R. Penner and J. Harer, Combinatorics of train tracks, Ann. of Math. Studies, 125, Princeton University Press, Princeton, NJ, 1992.

[Ker] S. Kerckhoff, The asymptotic geometry of Teichmller space, Topology 19 (1980), 23-41.

[Lu1] F. Luo, Geodesic length functions and Teichmüller spaces, Electron. Res. Announc. Amer. Math. Soc. 2 (1996), 34-41.

[Lu2] - Automorphisms of the complex of curves, preprint.

[Lu3] Simple loops on surfaces and their intersection numbers, preprint.

[MS] J. Morgan and P. Shalen, Valuations, trees, and degenerations of hyperbolic structures, I, Ann. of Math. 120 (1984), 401-476. 
[Pap] A. Papadopoulos, On Thurston's boundary of Teichmüller space and the extension of earthquakes, Topology Appl. 41 (1991), 147-177.

[Par] W. Parry, Axioms for translation length functions, Arboreal group theory (Berkeley, CA, 1988), Math. Sci. Res. Inst. Publ., 19, Springer, New York, 1991, pp. 295-330.

[Sc] L. Schneps, The Grothendieck theory of dessins d'enfants, Cambridge University Press, Cambridge, New York, 1994.

[Sk] R. Skora, Splittings of surfaces, J. Amer. Math. Soc. 9 (1996), 605-616.

[Th] W. Thurston, On the geometry and dynamics of diffeomorphisms of surfaces, Bull. Amer. Math. Soc. 19 (1988), 417-438.

Dept. of Math., Rutgers University, New Brunswick, NJ 08903

E-mail address: fluo@math.rutgers.edu 